\title{
Why the 18.6 year tide cannot explain the change of sign observed in $\dot{J}_{2}$
}

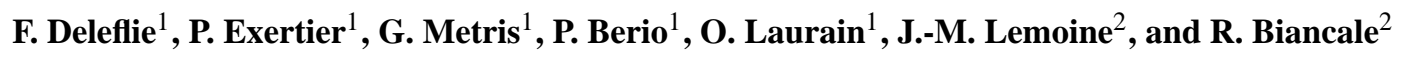 \\ ${ }^{1}$ Observatoire de la Côte d'Azur - CERGA/GRGS, Av. N. Copernic F-06130 Grasse, France \\ ${ }^{2}$ Observatoire Midi-Pyrénées OMP/GRGS, Av. E. Belin, F-31400 Toulouse, France
}

\begin{abstract}
Recent studies show a change, starting in 1998, in the behavior of the variation of the dynamical flattening of the Earth $\left(J_{2}\right)$, supposed to be constant (secular), and mainly due to the post glacial rebound effect.

In this paper, we study to what extent this behavior can be correlated or not with the 18.6 year tide: with more than twenty years of tracking data on LAGEOS-1, that is to say more than a period of 18.6 years, this effect can now be separated from the secular variation.

We use our theory of mean orbital motion, dedicated to studies of the long period effects on the orbital motion. We build one single arc of LAGEOS-1 from 1980 to 2002, which provides a continuous description of the orbital parameters. This is the great originality of our approach.

We focus our attention on the ascending node of LAGEOS-1, and we show that the change observed in $\dot{J}_{2}$ cannot be attributed to a statistical error due to a correlation, in short arcs results, between the secular variation of $J_{2}$ and the 18.6 year tide. The proof is based on the adjustment of amplitudes and phases of the long period tides, and on the shape of the residuals.
\end{abstract}

Key words. secular variation of $J_{2}, 18.6$ year tide, mean orbital motion

\section{Introduction}

Geodynamical parameters and in particular $J 2=-C_{2,0}$, characterizing the dynamical flattening of the Earth, contain a static part and a part varying with time with a large spectrum of periods: from around 10000 years for the post glacial rebound to some hours for atmospheric phenomena.

Among many contributions to the variable part of $C_{2,0}$ from solid Earth, Atmosphere and Ocean, there are long periodic ocean tides at 18.6 and 9.3 years. Although their amplitude and period have been fixed at some nominal values

Correspondence to: F. Deleflie

(Florent.Deleflie@obs-azur.fr)

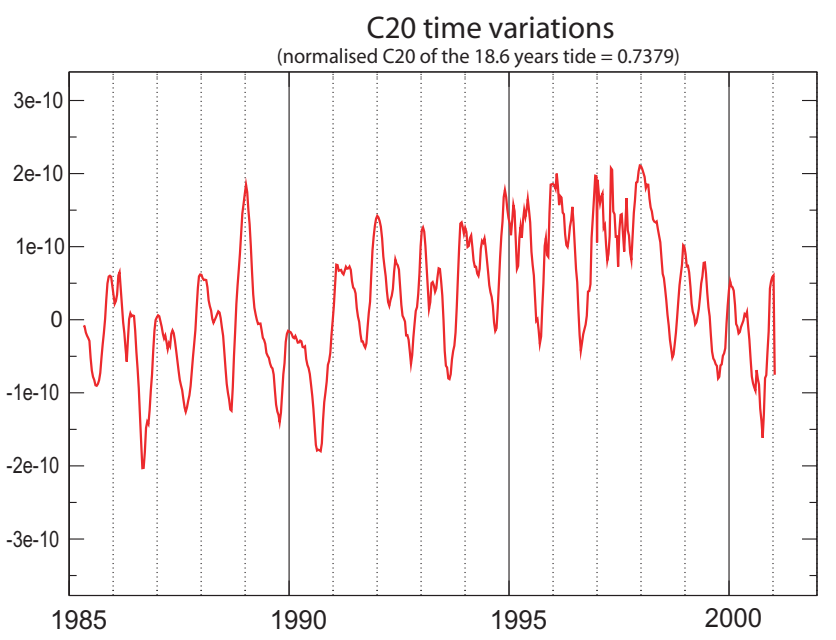

Fig. 1. Variations of $C_{20}$, deduced from short arc computations. These variations include seasonal variations others than the ones due to the atmospheric pressure. The values shown here are normalized, in opposition to all the other ones given hereafter.

considering the equilibrium hypothesis (Ray and Cartwright, 1994), slightly different values appear when considering inelastic phenomena (Zhu et al., 1996). On the other hand, $C_{2,0}$ variations also include seasonal variations mainly due to the displacement of the geophysical fluids at the surface of the Earth (Cazenave and Nerem, 2002). For example, variations in the global atmospheric pressure field leads to such annual and semi-annual effects, especially for the degree 2 of the field (Chao and Eanes, 1995).

Considering the fact that $\mathrm{J} 2$ is a global scale parameter, the most efficient method used to estimate its value (constant and variable parts) has been and is still based on satellite orbit perturbation analysis. As an inverse method, which is based on the dynamics of Earth artificial satellites, its principle consists in measuring subtle satellite orbit changes due to the influence of such geodynamical parameters. Obviously, these changes are all the more difficult to detect as it is the 

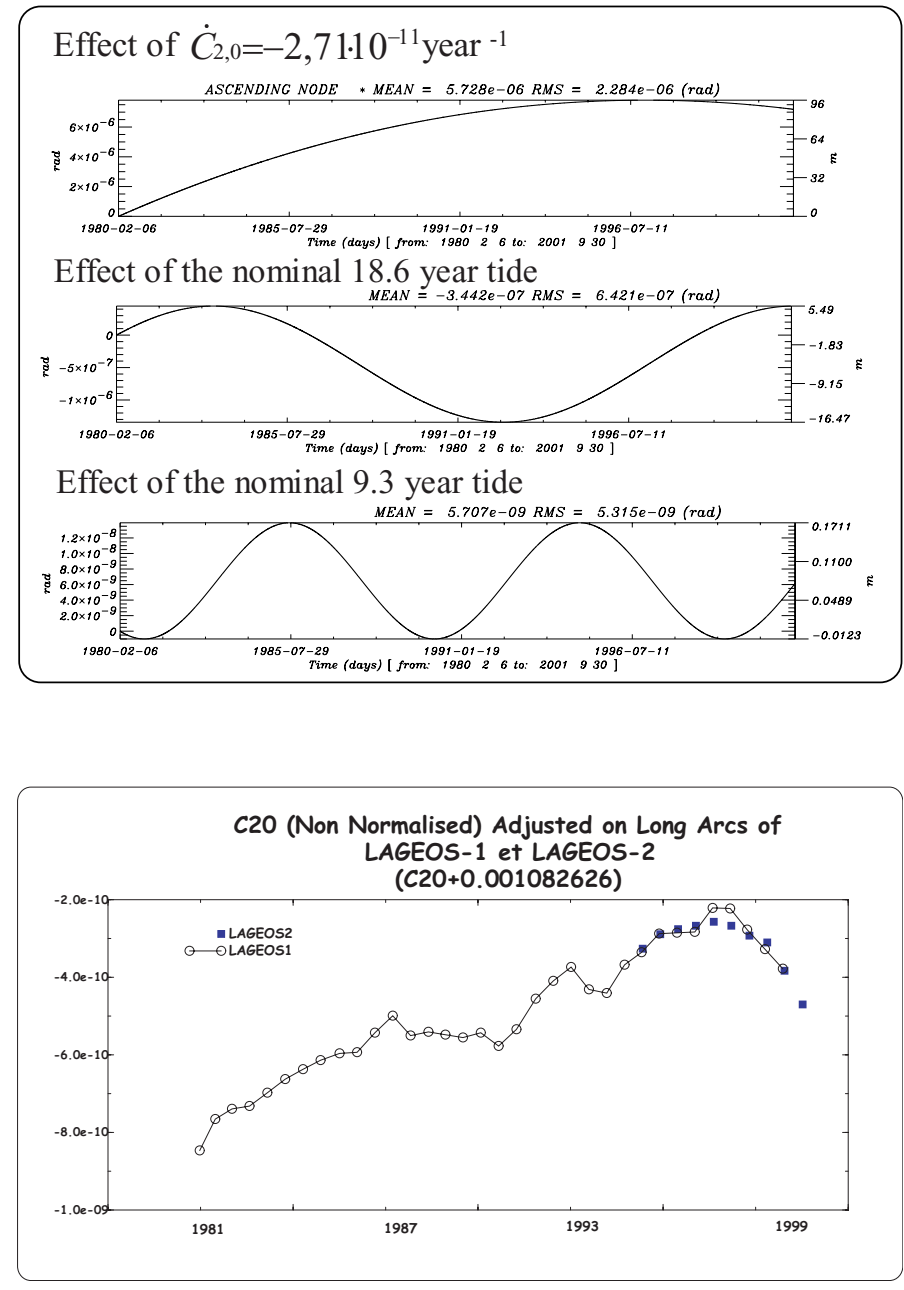

Fig. 2. Effects of geodynamical parameters on the ascending node of LAGEOS-1. The method of mean orbital motion links geodynamical parameters to corresponding mean effects on orbits. In this figure are shown, for three given parameters, the evolution of the ascending node over a period of 20 years. Each curve is the difference of two extrapolations: the first curve enlightens the impact of the secular variation of $C_{2,0}$, the second of the 18.6 year equilibrium tide [Ray and Cartwright,1994] which is equivalent to $1,23 \mathrm{~cm}$ of water, the third of the 9.3 year tide which is equivalent to $0,02 \mathrm{~cm}$ of water.
Fig. 3. Values of $C_{2,0}$ adjusted on long arcs from 1980 to 2001 . Each point is obtained from the computation of a 5year arc of LAGEOS-1 or -2, sliding every half year. As a consequence, the points are not independent one from another. No seasonal variations can be observed. One can notice the great agreement between both satellites, computed independently. variability of the parameter with time which is studied. As an example, let us recall that the $\dot{C}_{2,0}$ to $C_{2,0}$ ratio is of the order of $10^{-7}$.

Thanks to the dramatic improvement of the precise orbit determination with LAGEOS and other geodetic satellites, $\mathrm{J} 2$ time-series have been computed largely (Cox and Chao, 2002). These series show an average drift which is more or less secular from the end of the 70's to 1998 (see Fig. 1), effect which has been linked to the post glacial rebound and to the possible melting of polar caps (Yoder et al., 1983; Gegout, 1991). But, since then, it displays a strange long period behavior (change of sign observed in $\dot{C}_{2,0}$ ), possibly correlated to oceanic effects or to the liquid core of the Earth (Cox and Chao, 2002).

Here, we assess the possible correlation between this unexpected behavior and possible uncertainties (phase and amplitude) in the long period ocean tides (18.6 and $9.3 \mathrm{y}$ ). The 18.6 year tide cannot be decorrelated from the post glacial rebound effect over a short period of time, even if several satellite orbits are analyzed together. As a mater of fact, the effects of degree 2 of ocean and solid Earth have the same signature over a short period (less than one year roughly). On the other hand, over a long period of time (several years), the ascending node of artificial satellites clearly has parabolic and long period signatures, respectively due to post glacial rebound $\left(\dot{C}_{2,0}\right.$ secular) and 18.6 year tide.

Thus, in order to compute long orbital arcs over a period of time of 18-20 years, we have developed a dedicated orbit analysis technique. This technique enables us to describe the dynamical evolution of the mean orbital motion (Métris and Exertier, 1995), to compute mean orbital elements from precise fitted short arcs (Exertier, 1990), and then to estimate geodynamical parameters linked with long periodic and secular perturbations (Exertier et al., 1999.

From the analysis of orbit signatures (e.g. variations of the mean ascending node), using the LAGEOS-1 data between 1980 and 2002, we show that the combination of post glacial rebound and 18.6 year tide effects can be properly separated.

This leads us to conclude that the change of sign observed in $\dot{C}_{2,0}$ is certainly due to other effects, like oceanic ones.

\section{The specificity of our study: the mean orbital motion}

To build a long arc, and to take advantage of our method, we first have computed dynamical short arcs (10 days) of 


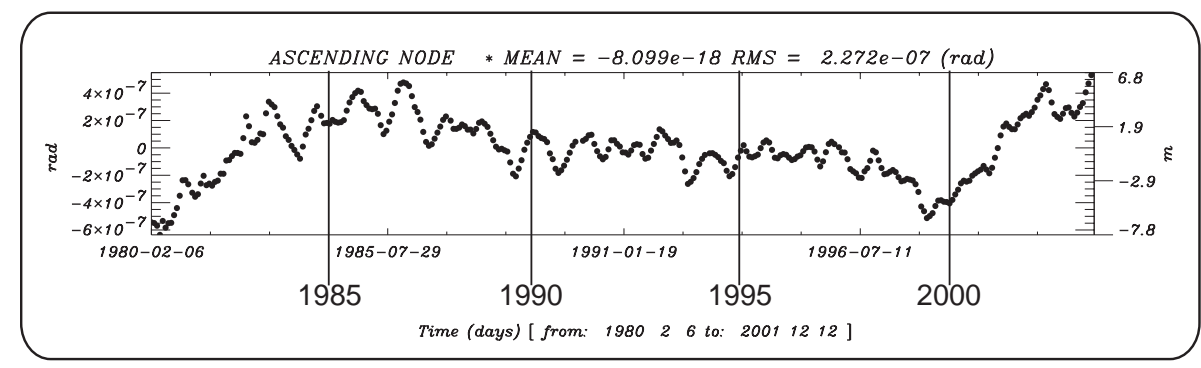

Fig. 4. What is observed on the ascending node. This figure shows the residuals on the ascending node of an arc computed from 1980 to 2002. There is no adjusted geodynamical parameter. Adjusted parameters: initial conditions, 5 empirical mean drag accelerations (varying from $-0,264410^{-11} \mathrm{~ms}^{-2}$ to $-0,484110^{-11} \mathrm{~ms}^{-2}$ ), a scale factor for the solar radiation pressure (equal to 0,95 ).

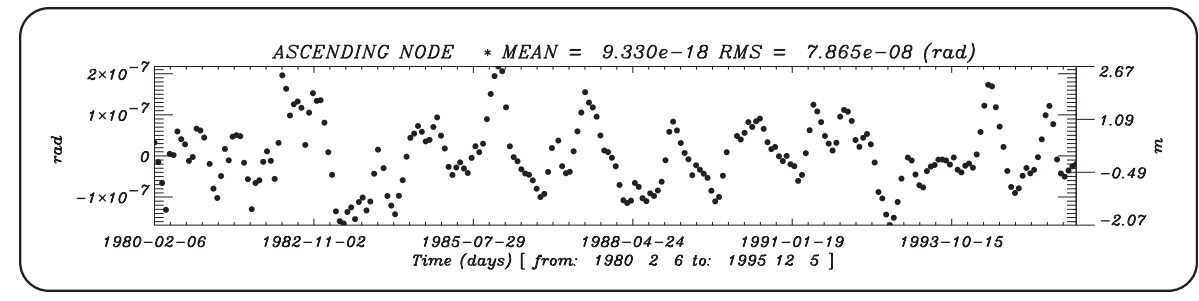

Fig. 5. Residuals on the ascending node of LAGEOS with an arc computed from 1980 to 1996 (Solution 1). Ajusted geodynamical parameters: 18.6 year tide, 9.3 year tide $C_{2,0}$ and $\dot{C}_{2,0}$. An annual and a semi-annual terms have also been adjusted.

LAGEOS-1, to provide information about the continuous behavior of the observed mean ascending node of the trajectories, and to get "mean observed elements". Then we have used our semi-analytical method (based on averaged perturbations and corresponding mean equations of motion; Métris and Exertier, 1995; Deleflie, 2002).

\subsection{Construction of "mean observed elements"}

There are two steps to obtain "observed mean elements" from measurements of geodetic techniques.

First, successive short arcs are computed with a classical numerical integration, over the whole period during which tracking data are available: we use the GINS software to compute 10-day arcs, on the basis of SLR data. Note that at this stage, only the state vector (and no physical parameter) is adjusted. These orbits are formulated with orbital elements and not with positions and velocities, in order to isolate the short from the long period perturbations.

Secondly, all the short period terms are removed, to get one single set of 6 mean orbital element every 10 days. This filtering approach, applied in the CANEL software, is described in (Exertier, 1990).

These "observed mean elements " are seen as observations of the mean motion, providing us the possibility to adjust orbit and geodynamical parameters.

\subsection{Interests of the semi-analytical theory}

The theoretical long arc orbits are adjusted on the basis of these observations obtained from CANEL, in the CODIOR software, making residual temporal series for each mean orbital element.

Our semi-analytical theory of the mean orbital motion is able to compute effects due to mean potentials (Earth, Moon and Sun, and planets) and mean surface forces (radiation pressures and drag) acting on a given satellite. The method has been built in a such way that sensibilities are computed together with the mean orbit itself.

As a result of orbital dynamics, the $C_{2,0}$ coefficient generates a precession of the ascending node of an orbit. Over long arcs, this secular motion is much more important than short periodic variations due to $C_{2,0}$. We can express $C_{2,0}$, seen as a parameter varying with time, such as:

$$
\begin{gathered}
C_{2,0}(t)=C_{2,0}\left(t_{0}\right)+\dot{C}_{2,0}\left(t-t_{0}\right)+a_{18,6} \cos \left(\omega_{18,6} t\right) \\
\quad+a_{9,3} \cos \left(\omega_{9,3}\right)+T_{A N}+T_{S A}
\end{gathered}
$$

where 18, 6 and 9, 3 denote respectively the quantities linked to the 18.6 and the 9.3 year tides. $T_{A N}$ is an annual term, $T_{S A}$ a semi-annual term.

Values of effective values of $C_{2,0}(t)$ are shown Fig. 3; There is no seasonal variation in $C_{2,0}(t)$ because they are adjusted directly in $T_{A N}$ and $T_{S A}$. These values have been obtained from long arcs of LAGEOS-1 and -2. LAGEOS-2 confirms the results obtained from LAGEOS-1, showing that the strong variations are not linked to the non-gravitational forces acting on LAGEOS-1 (Métris et al., 1998). Indeed, let us recall that satellite dynamics imposes strong correlations between the semi-major axis and the node. As a consequence, any residuals in the mean semi-major axis can have a corresponding signature in the mean ascending node. That's why empirical orbital coefficients are also estimated along 
Table 1. Adjusted zonal parameters

\begin{tabular}{llll}
\hline Arc & $\begin{array}{c}\text { Period of } \\
\text { the arc }\end{array}$ & \multicolumn{1}{l}{$C_{2,0}$} & $\dot{C}_{2,0}$ \\
& & & year \\
\hline 1 & $1980-1996$ & $-0,10826264310^{-2} \pm 0,110^{-9}$ & $2,7210^{-11} \pm 0,110^{-10}$ \\
2 & $1980-2002$ & $-0,10826263610^{-2} \pm 0,410^{-10}$ & $2,7010^{-11} \pm 0,910^{-12}$ \\
3 & $1980-2002$ & $-0,10826263610^{-2} \pm 0,410^{-10}$ & $2,8310^{-11} \pm 0,710^{-12}$
\end{tabular}

Table 2. Adjusted tidal parameters

\begin{tabular}{|c|c|c|c|c|c|c|c|c|c|}
\hline \multirow[t]{2}{*}{ Arc } & \multirow{2}{*}{$\begin{array}{l}\text { Period of } \\
\text { the arc }\end{array}$} & \multicolumn{4}{|c|}{18.6 year tide } & \multicolumn{4}{|c|}{9.3 year tide } \\
\hline & & $\begin{array}{l}\text { Ampl. } \\
\mathrm{cm}\end{array}$ & $\begin{array}{l}\text { Phase } \\
\text { deg }\end{array}$ & $\begin{array}{l}\mathrm{C} \\
\mathrm{cm}\end{array}$ & $\begin{array}{l}\mathrm{S} \\
\mathrm{cm}\end{array}$ & $\begin{array}{l}\text { Ampl. } \\
\mathrm{cm}\end{array}$ & $\begin{array}{l}\text { Phase } \\
\text { deg }\end{array}$ & $\begin{array}{l}\mathrm{C} \\
\mathrm{cm}\end{array}$ & $\begin{array}{l}\mathrm{S} \\
\mathrm{cm}\end{array}$ \\
\hline 1 & $1980-1996$ & 1,60 & 81 & $1,58 \pm 0,1$ & $0,27 \pm 0,5$ & 0,25 & -7 & $0,03 \pm 0,09$ & $-0,25 \pm 0,2$ \\
\hline 2 & $1980-2002$ & 1,45 & 77 & $1,41 \pm 0,05$ & $0,34 \pm 0,03$ & 0,49 & -50 & $0,37 \pm 0,05$ & $-0,32 \pm 0,04$ \\
\hline 3 & $1980-2002$ & 1,50 & 78 & $1,47 \pm 0,04$ & $0,31 \pm 0,03$ & - & - & - & - \\
\hline
\end{tabular}

the long arc, the mean surface forces being at present not perfectly and totally modeled.

Obviously, as a result of the analysis of a single arc of LAGEOS, the estimated geodynamical coefficient $C_{2,0}(t)$ is is an effective one. It contains, in addition to $C_{2,0}$ secular, the effect of the even harmonic coefficients of higher degree; the ascending node variations being the result of a linear combination of the variations of all even zonals (Eanes, 1995; Eanes and Bettadpur, 1996).

The ascending node is well adapted to study the specific signatures of each component of Eq. (1), because of the shape of the corresponding Lagrange equation:

$$
\begin{aligned}
\frac{d \Omega}{d t} & =\frac{1}{\sqrt{\mu a} \sqrt{1-e^{2}} \sin (i)} C_{2,0}(t) \frac{\mu}{a}\left(\frac{R_{0}}{a}\right)^{2} \\
& F_{2,0,1}^{\prime}(i) \sum_{q=-\infty}^{\infty} G_{2,1, q}(e) \cos \left(\psi\left(M, \omega, \Omega, T S_{G}\right)\right)
\end{aligned}
$$

where $U$ denotes the gravitational potential, and $\mu$ the gravitational constant of the Earth (Kaula, 1966). Since $C_{2,0}(t)$ is proportional to $\frac{d \Omega}{d t}$, and since the value of the orbital inclination of LAGEOS orbits is high, $\Omega$ is thus a very good variable to study the effect of $C_{2,0}(t)$.

Figure 2 shows the specific signatures on the node. Over a long period of time, it is thus possible to properly estimate the corresponding parameters (amplitude and phase) from the node. The present goal consists in checking that there is no value of the involved coefficients in Eq. (1) which can explain what is observed on the ascending node. In other words, we show that the strong variation observed in $C_{2,0}(t)$ has a geophysical origin, and is not due to statistic correlations (which can appear if the period of analysis is too short).

\section{Adjustment of the 18.6 year and the 9.3 year tides}

We have computed two long arcs using the following standards, the first from 1980 to 1996 and the second from 1980 to 2002:

- GRIM5-S1 for the gravity field model (Biancale et al., 2000),

- (Schwiderski, 1980) for the ocean tides,

- the Love numbers $k_{2}=0,299$ and $k_{3}=0,094$ to compute the terrestrial tides,

- the VSOP82 theory for the luni-solar effects and the planets (Bretagnon and Francou, 1988).

These two single long arcs are based on a nominal geodynamical model including long period tides. Figures 4, 5, 6, 7 show the residuals on the ascending node of the computed arcs in each case, adjusting or not some physical parameters.

Figure 4 shows the residuals from 1980 to 2002 when no geodynamical parameter is adjusted. One can notice a strange characteristic at the beginning of 1998. At this sage, it is impossible to say whether it is linked to an inappropriate value of $\dot{C}_{2,0}$ or not. Here, we have fixed $\dot{C}_{2,0}=$ $2,7110^{-11}$ year $^{-1}$, the 18.6 year tide to $1,23 \mathrm{~cm}$, and the 9,3 tide to $0,02 \mathrm{~cm}$. The other characteristics of the mean model are nominal.

To evaluate the impact of this strong behavior on the value of tidal parameters, we have computed an arc (Solution 1) from 1980 to 1996 (Fig. 5). The 18.6 and the 9.3 year tide are adjusted (Table 2), as well as $C_{2,0}\left(t_{0}\right)$ and $\dot{C}_{2,0}$ (Table 1).

Then the same computation is made from 1980 to 2002 (Solution 2: Fig. 6). Because of the unacceptable value of the 9.3 year tide (as well as in Solution 1) which can "absorb" 


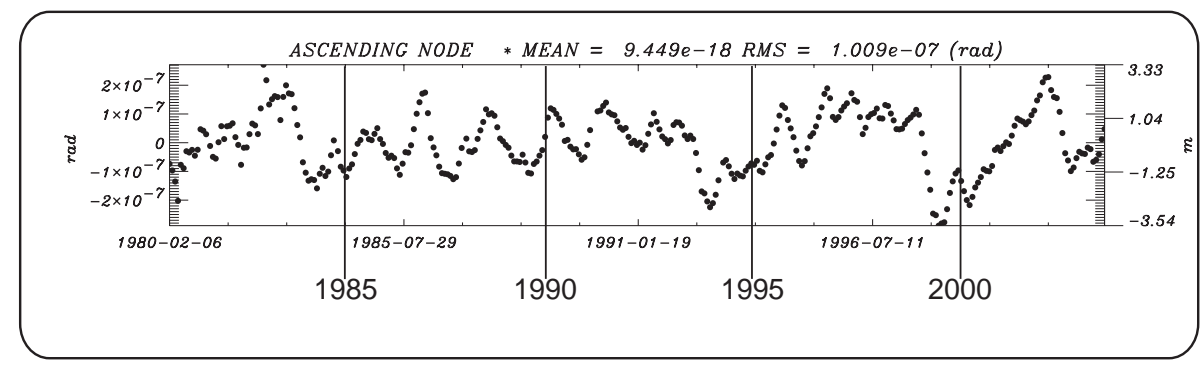

Fig. 6. Residuals on the ascending node of LAGEOS with an arc computed from 1980 to 2002, including the adjustment of the 9.3 year tide (Solution 2). Adjusted geodynamical parameters: 18.6 year tide, 9.3 year tide, $C_{2,0}$ and $\dot{C}_{2,0}$. An annual and a semi-annual terms have also been adjusted.

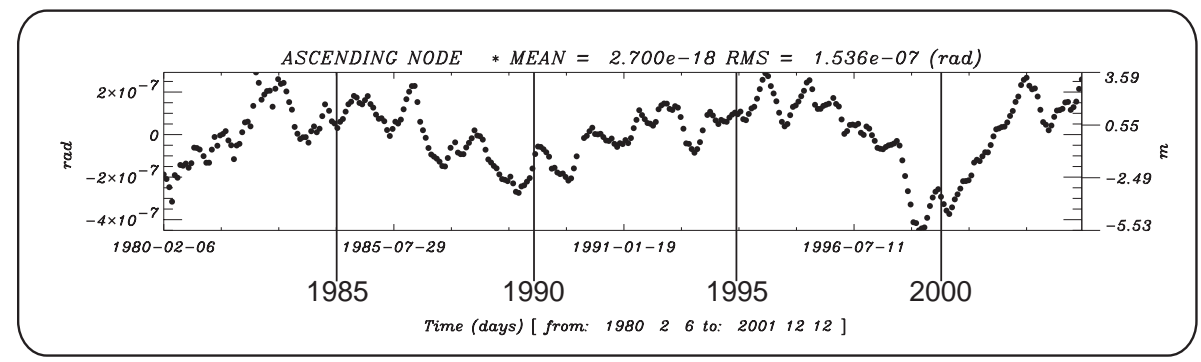

Fig. 7. Residuals on the ascending node of LAGEOS with an arc computed from 1980 to 2002, NOT including the adjustment of the 9.3 year tide (Solution 3). Adjusted geodynamical parameters: 18.6 year tide, $C_{2,0}$ and $\dot{C}_{2,0}$. An annual and a semi-annual terms have also been adjusted.

part of the 18.6 year signal, we have computed an arc from 1980 to 1996, without adjusting the 9.3 year tide (Solution 3). One can see in Fig. 7 with naked eye a signal at this period.

The adjusted values of $C_{2,0}\left(t_{0}\right)$ and $\dot{C}_{2,0}$ have a great stability, and are not much affected by the strong variation observed in the residuals (Solutions 2 and 3). Moreover, there are well decorrelated from the value of their amplitude of the 18.6 year tide (correlation equal to 0.25 in Solution 1 between $\dot{C}_{2,0}$ and $C_{18,6}$ ). Finally, the amplitude of the 18.6 year tide is also rather stable ; the difference of $0,1 \mathrm{~cm}$ between Solutions 1 and 3 cannot breed a difference in the corresponding signal of the ascending node larger than 2 meters.

We conclude that the behavior beginning in 1998 observed on Fig. 7 cannot be absorbed neither by the 18.6 year tide, nor by a purely secular variation of $J_{2}$. On the other hand, a 9.3 year tide can partly mimic the observed residual signal but the adjusted amplitude exceeds 20 times the commonly accepted value.

\section{Conclusion}

Our continuous temporal series of the ascending node confirms what is observed on the orbit of LAGEOS-1 with classical short arc technique: something happened at the beginning of 1998 in the orbital motion. Our long period approach makes us conclude that this phenomenon cannot be related to the post-glacial rebound or to the 18.6 year tide, since these phenomena have specific signatures on the orbital motion which do not correspond to what is effectively observed. Is it the same phenomenon which triggered the two changes in the sign of $\dot{C}_{2,0}$ which can be seen in the Fig. 3 at the end of 1989 and at the end of 1985 ? Another study, with different satellites and more geodynamical adjusted parameters would be interesting.

\section{References}

Biancale, R., Balmino, G., Lemoine, J.-M., Marty, J.-C., Moynot, B., Barlier, F., Exertier, P., Laurain, O., and Gegout, P.: A new global Earth's gravity field model from satellite orbit perturbations: GRIM5-S1, Geophys. Res. Lett., 27, 22, 3611-3614, 2000.

Bretagnon, P. and Francou, G.: Planetary theories in rectangular and spherical variables. VSOP87 solutions, Astron. Astroph., 202, 309-315, 1988.

Bruinsma, S. L.: Semi-analytical long-arc satellite orbit computation and the estimation of time-varying gravity parameters, Phd Thesis, Delft University of Tech., Delft, Pays-Bas, 2002.

Cazenave, A. and Nerem, R. S.: Redistributing Earth's Mass, Science, 297, 783-784, 2002.

Chao, B. F. and Eanes, R.: Global gravitational changes due to atmospheric mass redistribution as observed by the Lageos nodal residual, Geophys. J. Int., 122, 755-764, 1995.

Cox, C. M. and CHao, B. F.: Detection of a Large-Scale Mass Redistribution in the Terrestrial System Since 1998, Science, 297, 831-833, 2002. 
Deleflie, F.: Théorie Semi-Analytique des Mouvements QuasiCirculaires Moyens en Mécanique Spatiale - Applications aux Satellites Géodésiques, Thèse de doctorat de l'Observatoire de Paris, 2002.

Deprit, A.: Canonical transformations depending on a small parameter, Celest. Mech. 1, 12-30, 1969.

Eanes, R. J.: A study of temporal variations in Earth's gravitational field using LAGEOS-1 laser range observations, Ph.D. Thesis CSR-95-8, The University of Texas at Austin, 1995.

Eanes, R. J. and Bettadpur, S.: in: "Global Gravity Field and Its Temporal Variations”, pp. 30-41, Springer-Verlag, New York, 1996.

Enfield, D. B.: El Niño, past and present, Rev. Geophys., 27, 1, 158-187, 1989.

Exertier, P.: Precise determination of mean orbital elements from osculating elements, by semi-analytical filtering, manuscripta geodaetica, 15, 115-123, 1990.

Exertier, P., Bruinsma, S., Métris, G., Boudon, Y., and Barlier, F.: Geodynamics from the analysis of the mean orbital motion of geodetic satellites, Int. Assoc. of Geodesy Symposia, 121, 262270, Berlin, 2000.

Gegout, P.: Variations temporelles saisonnières et séculaires des premiers termes zonaux du champ de gravité de la Terre, Diplôme d'ingénieur de l'Ecole de Physique du Globe, Univer- sité Louis Pasteur, Strasbourg, 1991.

Kaula, W. M.: Theory of satellite geodesy, Blaisdell Publishing Company, Waltham, Masachusetts, 1966.

Métris, G. and Exertier, P.: Semi-analytical Theory of the Mean Orbital Motion, Astron. Astrophys., 294, 278-286, 1995.

Métris, G., Vokroulický, D., Ries, J. C., and Eanes, R. J.: LAGEOS spin axis and nongravitational excitations of its orbit, COSPAR meeting, Nagoya, Japan, 1998.

Ray, R. D. and Cartwright, D. E.: Satellite altimeter observations of the Mf and Mm ocean tides, with simultaneous orbit corrections, in: Gravimetry and Space Techniques Applied to Geodynamics and Ocean Dynamics, (Eds) Schutz, B. E., Anderson, A., Froidevaux, C., and Parke, M., 69-78, AGU Geophyical Monograph 82, IUGG Vol. 17, Washington D.C., 1994.

Schwiderski, E. W.: Ocean tides, Part 1 : Global ocean tidal equations, Marine Geodesy, 3, 161-207, 1980.

Yoder, C. F., Milliams, J. G., Dickey, J. D., Schutz, B. E., Eanes, R. J., and Tapley, B. D.: Secular variation of Earth's gravitational harmonics $\mathrm{J} 2$ coefficient from LAGEOS and non tidal acceleration of Earth rotation, Nature, 303, 757-762, 1983.

Zhu, Y., Shum, C. K., Cheng, M. K., Tapley, B. D., and Chao, B. F.: Long-period variations in gravity field caused by mantle anelasticity, J. Geophys. Res., 101, B.5, 11 243-11 248, 1996. 